\title{
Adopción de tecnologías y prácticas agropecuarias en sistemas de producción en Jinotega, Nicaragua
}

\section{Adoption of technologies and practices in agricultural production systems in Jinotega, Nicaragua}

\section{Eddin David Pérez Medal' ${ }^{1}$, Roberto Carlos Larios-González ${ }^{2}$}

${ }^{1}$ MSc. en innovación agropecuaria, Instituto Nicaragüense de Tecnología Agropecuaria, ORCID: https://orcid.org/0000-0003-2668-4378 / edypm2007@yahoo.es / MSc. en agroecología y desarrollo sostenible, Universidad Nacional Agraria, ORCID: https://orcid.org/0000-00034290-2216 / roberto.larios@ci.una.edu.ni

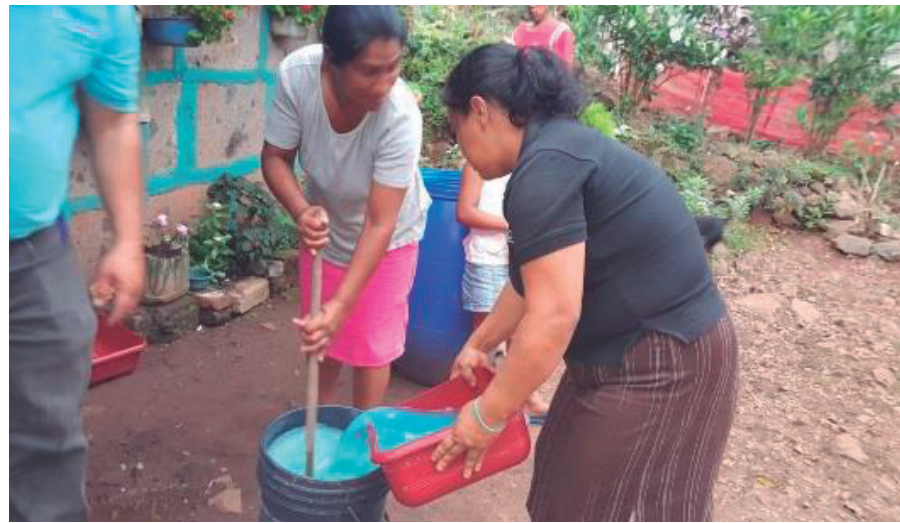

\section{RESUMEN}

La adopción de prácticas y tecnologías agropecuarias está determinada por las condiciones socio-económicos del productor y por las características de los sistemas de producción. El objetivo principal fue identificar factores y actividades de extensión que favorecen la adopción de prácticas y tecnologías agropecuarias. Esta investigación se realizó en siete municipios del departamento de Jinotega, ubicado en el norte de Nicaragua, Centro América. El tamaño de la muestra se determinó a través del cálculo de poblaciones finitas de un universo de 1470 pequeños productores, quienes han trabajado en colaboración con el Instituto Nicaragüense de Tecnología Agropecuaria, universidades y otras instituciones dedicadas al desarrollo de sector agrario y que han sido parte de procesos de extensión e investigación participativa. Las variables evaluadas fueron nivel de escolaridad y edad del productor, número de hijos, tenencia de la tierra, capacitaciones agropecuarias recibidas y métodos de difusión de tecnologías; todas ellas en relación al nivel de aplicación y adopción de prácticas y tecnologías. Para el registro de la información se utilizaron encuestas semiestructuradas, observación in situ y grupo focal. Los factores socioeconómicos, así como el acompañamiento técnico y los mecanismos de difusión de las tecnologías, son elementos determinantes en la aplicación y adopción de prácticas y tecnologías agropecuarias.

Palabras clave: factores socioeconómicos, extensión, difusión de tecnologías, capacitaciones agropecuarias.

Abreviatura: INTA, Instituto Nicaragüense de Tecnología Agropecuaría.
The adoption of agricultural technologies and practices is determined by the socio-economic conditions and characteristics of production systems. The main aim was to identify factors and extension activities that facilitate adoption of agricultural technologies and practices. This research was conducted in seven municipalities at Department of Jinotega, located in Northern Nicaragua, Central America. The sample size was determined by calculating finite populations of a universe of 1470 small scale producers, those who have worked in collaboration with the Nicaraguan Institute of Agricultural Technology, universities and other institutions which have been dedicated to the development of agricultural sector and which have been part of processes of participatory research and extension. The evaluated variables were producer's age and education level, number of children, land's ownership, in how many agricultural training and technology dissemination methods has been involved; all of them in relation to the level of implementation and adoption of technologies and practices. Semi-structured surveys, on-site observation and focus group were used for registration information. Socio-economic factors, as well as the technical support and mechanisms for the dissemination of technologies, are key elements in the implementation and adoption of agricultural technologies and practices.

Key words: socio-economic factors, extension and diffusion of technologies, agricultural training.
Recibido: 5 de febrero del 2018 Aceptado: 15 de junio del 2018

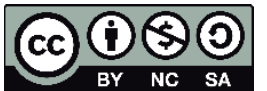

Los artículos de la revista La Calera de la Universidad Nacional Agraria, Nicaragua, se comparten bajo términos de la licencia Creative Commons: Reconocimiento, No Comercial, Compartir Igual. Las autorizaciones adicionales a las aquí delimitadas se pueden obtener en el correo freddy.aleman@ci.una.edu.ni

C Copyright 2018. Universidad Nacional Agraria 


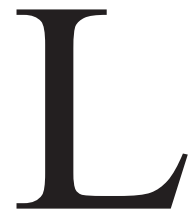

a adopción de tecnologías y prácticas agropecuarias es fundamental para mejorar la productividad, diversificar los sistemas de producción, conservar los recursos naturales y mejorar el nivel de vida de las familias productoras.

En el departamento de Jinotega se registran aproximadamente 30330 productores agropecuarios, con un total de 467621 hectáreas, siendo sus principales rubros el café, los granos básicos (maíz, frijol y sorgo), hortalizas y la ganadería bovina (MAG, 2012).

Según INTA (2015), en Nicaragua existen 284 tecnologías agropecuarias, de las cuales 231 han sido generadas por esta institución y 53 tecnologías por universidades relacionadas al sector agropecuario y los recursos naturales, algunas de ellas, adoptadas por las familias productoras de acuerdo a las condiciones agroclimáticas, por su eficiencia, bajo costo y fácil aplicación.

Debido a que Jinotega tiene una contribución importante en la producción nacional, este estudio plantea un análisis de la situación de la adopción tecnológica en siete de sus ocho municipios, así como la identificación de factores que la afectan o favorecen. Se consideran algunos aspectos socioeconómicos como la escolaridad y edad del productor, número de hijos que habitan en la finca y tenencia de la tierra; además, se detalla la adopción de tecnologías y prácticas, las capacitaciones recibidas y los métodos de extensión utilizados para la difusión e implementación de las tecnologías y prácticas agropecuarias.

Según Rojas (2015), en Nicaragua debemos medir de manera permanente el nivel de adopción e innovación de las tecnologías, proceso planteado en el Sistema Nicaragüense de Investigación e Innovación Agropecuaria (SINIA). Este sistema fue conformado en el 2015, como un mecanismo de concertación, coordinación, planificación, implementación, seguimiento y evaluación de la actividad de investigación e innovación agropecuaria del país.

El propósito de este estudio fue identificar los factores que determinan la adopción de tecnologías y prácticas agropecuarias en sistemas de producción de pequeños productores de Jinotega, las capacitaciones recibidas por la familia productora y los métodos de difusión de las tecnologías que permiten una mayor aplicación y adopción.

\section{MATERIALES Y MÉTODOS}

Localización y descripción del área de estudio. Este trabajo se realizó en siete municipios del departamento de Jinotega (Jinotega, La Concordia, San Rafael del Norte, San Sebastián de Yalí, Santa María de Pantasma, El Cuá y
Wiwili) durante el periodo de julio a diciembre del 2016. El departamento de Jinotega se encuentra ubicado a 161 km de Managua, capital de Nicaragua, y se destaca por ser el principal productor de café en el país y el segundo en granos básicos, principalmente en la producción de maíz y frijol. La producción ganadera extensiva de doble propósito es otra actividad fundamental, así como de hortalizas y frutas (Gamboa y Vanderschaeghe, 2013).

Diseño metodológico. Esta investigación es cuantitativa no experimental; de tipo descriptiva, expofacto, longitudinal y de corte transversal ya que se describen los acontecimientos, es expofacto porque se analizaron hechos ocurridos antes del estudio, haciendo alusión a que primero se produce el hecho y después se analizan las posibles causas y consecuencias, por lo que se trata de un tipo de investigación en donde no se modifica el fenómeno o situación objeto de análisis (Bernardo y Caldero, 2000). Longitudinal porque buscan analizar cambios o continuidad en las características de los sujetos que se estudian y de corte transversal porque se estudió la incidencia del paso del tiempo en el desarrollo de los sujetos de diferentes edades de forma que cubran el arco del tiempo propuesto en el estudio.

Se consideró como universo de estudios a 1470 productores del departamento de Jinotega, quienes han trabajado en colaboración con el Instituto Nicaragüense de Tecnología Agropecuaria (INTA) y han sido parte de proceso de extensión agropecuaria, procesos de investigación participativa y procesos de transferencia tecnológicas, recibiendo capacitaciones en temas agropecuarios.

Para la definición de la muestra se utilizó la fórmula propuesta por Münch y Ángeles (1996), que se utiliza para el cálculo de poblaciones finitas, cuando conocemos el total de la población o universo de estudio. En este caso, 65 productores. Su cálculo se base en la ecuación:

$$
\mathrm{n}=\frac{\mathrm{Z}^{2} * \mathrm{p} * \mathrm{q}^{*} \mathrm{~N}}{\left(\mathrm{~N}^{*} \mathrm{e}^{2}\right)+\left(\mathrm{Z}^{2} * \mathrm{p} * \mathrm{q}\right)}
$$

Dónde:

$\mathrm{n}=$ Tamaño de la muestra resultante, $\mathrm{Z}=$ Nivel de confianza. En este estudio el nivel de confianza es igual a $90 \%$, el valor de $\mathrm{Z}$ correspondiente $=2.74, \mathrm{p}$ y $\mathrm{q}=$ probabilidades complementarias e igual a $0.5, \mathrm{~N}=1470$ corresponde al universo, e $=$ Error de estimación aceptable para encuestas $10 \%$.

También se consideraron a los técnicos de la oficina de INTA en Jinotega, quienes estuvieron involucrados en procesos de investigación y extensión en los municipios en estudio. 
Herramientas y técnicas para el registro de información. Para el registro de la información se utilizaron encuestas semi estructuradas, observación in situ y grupo focal. Las encuestas semi estructuradas fueron aplicadas a los 65 productores y a los ocho técnicos del INTA; se realizaron visitas en cada sistema de producción y en el caso de los técnicos del INTA, se trabajó en las oficinas de la delegación departamental de Jinotega, ubicada en el municipio de San Rafael del Norte.

Durante el levantamiento de la información, se integraron a ocho estudiantes de la carrera de agroecológica que oferta la Universidad Nacional Autónoma de Nicaragua (UNAN-FAREM-Jinotega), estrategia del trabajo que se desarrolla en el Consejo Regional de Investigación e Innovación Agropecuaria de la VI Región, en el marco del Sistema Nicaragüense de Investigación e Innovación Agropecuaria (SNIA).

En otro momento se trabajó con un grupo focal conformado por 20 productores, seleccionados según los siguientes criterios: que representaran a los siete municipios, proximidad con la oficina del INTA (lugar donde se realizó la reunión con los productores), haber participado en actividades de extensión organizadas por el INTA, y productores que han sido parte de las capacitaciones realizadas por el INTA.

La observación in situ tuvo el propósito de lograr un acercamiento con la familia productora, ubicarse físicamente y registrar características de los sistemas de producción, así como identificar la aplicación de prácticas y tecnologías en los sistemas de producción.

\section{Variables evaluadas}

Factores determinantes en la adopción de prácticas y tecnologías. Se estudiaron algunos factores que interviene en la adopción de tecnologías agropecuarias, entre ellos el nivel de escolaridad, edad del productor, número de hijos $\mathrm{y}$ tenencia de la tierra.

Adopción de tecnologías y prácticas agropecuarias. Se identificó la adopción de las tecnologías y prácticas agrícolas y pecuarias promovidas por el INTA.

Capacitaciones agropecuarias recibidas. Se identificaron las temáticas de capacitación facilitadas por el INTA y los métodos de difusión de las tecnologías y prácticas utilizados.

Análisis de la información. Los datos cuantitativos provenientes de las encuestas semiestructuradas se organizaron en hojas de Excel para categorizarlas y agruparlas, y se analizaron con el programa SPSS versión 20.0. Los resultados se presentan de forma descriptiva usando figuras y cuadros.
Etapas de la investigación. El proceso de investigación se dividió en cinco fases. Fase 1: revisión de base de datos sobre productores organizados por el INTA en el programa de promotoría rural (1 470 productores), en la fase 2, se elaboraron las encuestas y se trabajó con los estudiantes para explicarles el objetivo del estudio y capacitarles en la aplicación de estas. Posteriormente se realizó la validación del instrumento con productores del municipio de Jinotega, debido a la cercanía y a la diversidad productiva que se presenta. Esta validación permitió el diseño final del instrumento. La fase 3 consistió en la visita y aplicación de las encuestas a los 65 productores seleccionados en los siete municipios; posteriormente se realizó el llenado de las encuestas con los técnicos de la oficina de INTA delegación Jinotega. En la fase 4, según los criterios de selección, se convocaron a 20 productores para conformar un grupo focal, usando una guía de preguntas orientadoras para ampliar la información sobre los factores que favorecen o limitan la aplicación de tecnologías agropecuarias, en los sistemas de producción. La fase 5 estuvo orientada al análisis e interpretación de los datos, se categorizaron, agruparon y se analizaron, utilizando los programas de Excel y SPSS 20.0.

\section{RESULTADOS Y DISCUSIÓN Factores determinantes en la adopción de prácticas y tecnologías}

Nivel de escolaridad de los productores. Se registra que la mayoría de los productores cuentan con un nivel de educación primaria (52\%) y secundaria $(20 \%)$, y existe un $10 \%$ a nivel de técnico agropecuario y un $18 \%$ alfabetizados.

Greig (2009) plantea que el nivel educativo del agricultor se considera como una proximidad al acceso de la información, y se espera una relación directa entre el nivel educativo y la adopción de tecnología; esto se evidencia en las comunidades de Jinotega, ya que los productores con nivel de educación secundaria y técnica han aceptado y aplicado entre seis y 16 tecnologías y prácticas agropecuarias, mientras que los productores con educación primaria entre seis y 10 , y los alfabetizados menor cantidad (3-10).

Según Pineda y Guzmán (2015) las personas con baja escolaridad presentan mayores dificultades en la asimilación de la información y cuentan con menos herramientas que les permitan apropiarse de la información y decidir la adopción de tecnologías.

Edad del productor. El $63 \%$ de los productores se ubican entre las edades de 45 a 75 años y el $37 \%$ entre 25 y 45 . 
Según Roco et al., (2012) la edad del productor tiene una influencia positiva en la aplicación y adopción de prácticas y tecnologías, y tiene relación con la naturaleza de la tecnología, al asociarse con agricultura de conservación, y la baja disponibilidad de insumos agrícolas.

En el caso de los productores de Jinotega, la aplicación y adopción de tecnologías y prácticas agropecuarias es más efectiva en aquellos con edades menores de 45 años, ya que demuestran interés en el uso de nuevos modelos productivos en las fincas; en cambio, productores mayores a esta edad, hacen menos uso porque se limitan más al conocimiento empírico adquirido a través de los años y al manejo tradicional de su finca. Al relacionar la escolaridad, la edad y la adopción de tecnologías se registra que, a menor edad, mayor escolaridad y mayor aplicación y adopción de prácticas y tecnologías agropecuarias.

Número de hijos. La disponibilidad de mano de obra familiar es un aspecto importante porque determina en muchos casos la aplicación y adopción de tecnología y la productividad de la finca. En este caso, el $49 \%$ de los productores tienen entre dos y cuatro hijos, con un rango de edad entre 10 y18 años, el 37\% tienen entre cuatro y 10 hijos y el $14 \%$ dos o ningún hijo. Todos los productores reportan que sus hijos aportan con la mano de obra familiar en mayor o menor medida, en relación a su edad. Esto último es respaldado por la FAO (2014), quien señala que la agricultura familiar es una forma de organizar la producción agrícola y es gestionada y dirigida por una familia que en su mayor parte depende de la mano de obra familiar, tanto de mujeres como de hombres.

En estos municipios de Jinotega, la adopción de tecnologías y prácticas es mayor en los sistemas de producción cuyas familias tiene mayor número de hijos que habitan en la finca, ya que, para la implementación de las tecnologías y la diversificación del sistema productivo, se demanda mucha mano de obra. Roco et al., (2012) plantean que se puede esperar que los productores con familias más numerosas posean mayor disponibilidad de mano de obra para adoptar tecnologías, aunque por el tipo de tecnología, el requerimiento de mano de obra es puntual en el tiempo.

Tenencia de la tierra. Según la FAO (2003), la tenencia de la tierra se puede referir a la gestión de la propiedad como el derecho a decidir la forma más indicada de utilizar la tierra, en particular, qué cultivos se van a producir y la manera de beneficiarse económicamente de la venta de los cultivos.

Se determinó que el $94 \%$ de los productores tienen título de propiedad, y son ellos los que hacen uso de un mayor número de prácticas y tecnologías, en algunos casos hasta un total de 17. Se registra también que un pequeño porcentaje de productores (3.7), alquilan la tierra para cultivarla, y se reportan entre cinco y seis tecnologías adoptadas. Esta condición podría deberse por disposición de los dueños de la tierra como un requisito de arrendamiento. También están los casos de las tierras heredadas, pero sin título de propiedad, donde se emplean hasta ocho tecnologías agropecuarias.

Si la tierra es propia, se puede disponer de los recursos, condición que permite decidir aplicar y adoptar en mayor medida las tecnologías y prácticas agropecuarias. Roco et al., (2012) plantean que la seguridad en la tenencia de la tierra tiene un efecto positivo en la adopción de tecnologías, ya que esto representa mayor capital y existe disposición a invertir en el largo plazo para la mejora de las fincas.

Adopción de prácticas agropecuarias. Las prácticas agropecuarias son definidas por el INTA (2016), como el conjunto de procedimientos y recomendaciones técnicas aplicables de forma sistemática en la producción agropecuaria; estas pueden ser labranza, siembra directa, podas, rotación de cultivos, ensilaje, henificación, tipos de cosecha, fertilización, entre otros.

En el cuadro 1 se detalla el porcentaje de productores por municipio que han adoptado las prácticas identificadas; por ejemplo, en Jinotega el 100\% de los productores utilizan barreras vivas, pero de ese $100 \%$, el $75 \%$ también incorporan rastrojos y establecen barreras muertas; y el $62 \%$ hacen uso de rotación de cultivos, desparasitación, vacunación y vitaminización.

La adopción de prácticas agropecuarias en estos sistemas de producción ocurre en mayor o menor porcentaje, y está en relación directa con los factores que determinan su adopción, lo que fueron descritos anteriormente. Las prácticas de mayor adopción corresponden a: barreras vivas, incorporación de rastrojos, desparasitación, vacunación, vitaminización, la no quema, rotación de cultivos y barreras muertas.

El uso de barreras vivas, barreras muertas y acequias son utilizadas como estrategias de conservación de suelo y agua; la no quema y la rotación de cultivos como estrategia del manejo integrado del suelo; el uso de neem más hoja de madero negro como bioinsumos para el manejo de plagas; y la desparasitación, vacunación, vitaminización y ordeño limpio como parte del manejo zoosanitarias. También se hace uso de la prueba de la sal para el manejo post cosecha; distancia de siembra en granos básicos para el manejo de enfermedades, y reforestación para el manejo del recurso hídrico. 
Cuadro 1. Practicas agropecuarias adoptadas en sistemas productivos de siete municipios de Jinotega

\begin{tabular}{|c|c|c|c|c|c|c|c|}
\hline \multirow{2}{*}{ Práctica* } & \multicolumn{7}{|c|}{ Porcentaje de productores por municipio } \\
\hline & Jinotega & $\begin{array}{c}\text { San Rafael } \\
\text { del Norte }\end{array}$ & $\begin{array}{c}\mathrm{La} \\
\text { Concordia } \\
\end{array}$ & Yalí** & Pantasma** & El Cuá & Wiwil \\
\hline Barreras vivas & 100 & 73 & 78 & 82 & 75 & 75 & 33 \\
\hline $\begin{array}{l}\text { Incorporación de } \\
\text { rastrojo }\end{array}$ & 75 & 64 & 67 & 82 & 62 & 75 & 33 \\
\hline Barreras muertas & 75 & 27 & 78 & 45 & 37 & 25 & 55 \\
\hline No quema & 25 & 73 & 36 & 73 & 50 & 37 & 44 \\
\hline Rotación de cultivo & 62 & 18 & 55 & 54 & 13 & 37 & 33 \\
\hline Cercas vivas & 37 & 45 & 0 & 18 & 37 & 50 & 44 \\
\hline Reforestación & 13 & 9 & 36 & 18 & 25 & 25 & 11 \\
\hline Distancia de siembra & 25 & 18 & 22 & 45 & 13 & 37 & 33 \\
\hline Trampas amarilla & 25 & 18 & 0 & 9 & 13 & 0 & 33 \\
\hline Insecticidas botánicos & 13 & 0 & 22 & 27 & 25 & 0 & 0 \\
\hline Prueba de la sal & 25 & 54 & 36 & 45 & 25 & 50 & 44 \\
\hline Tentaleo & 0 & 9 & 22 & 27 & 13 & 25 & 33 \\
\hline Ensilaje & 25 & 18 & 33 & 27 & 25 & 25 & 44 \\
\hline Concentrado caseros & 13 & 9 & 22 & 18 & 0 & 25 & 0 \\
\hline Desparasitación & 62 & 73 & 67 & 73 & 75 & 87 & 55 \\
\hline Ordeño limpio & 25 & 27 & 22 & 36 & 37 & 37 & 44 \\
\hline Vacunación & 62 & 54 & 55 & 64 & 50 & 37 & 67 \\
\hline Vitaminización & 62 & 54 & 55 & 64 & 50 & 37 & 67 \\
\hline
\end{tabular}

* Prácticas agropecuarias según INTA (2016) / ** Yalí: San Sebastián de Yalí, Pantasma: Santa María de Pantasma.

El fomento de prácticas de conservación y el uso adecuado de los recursos en los sistemas de producción, sobre la base de los principios agroecológicos, es importante para el desarrollo de la agricultura; estos principios son puestos en práctica por instituciones como el INTA y la Universidad Nacional Agraria (UNA) en conjunto en conjunto con los agricultores a través de la investigación participativa y procesos de extensión, como estrategia para contribuir con la seguridad alimentaria y nutricional, enfrentar los efectos del cambio climático y transitar hacia una agricultura sostenible.

Según la FAO (1998), el desarrollo agrícola sostenible involucra el manejo y la conservación de los recursos naturales, de manera que se asegure la satisfacción permanente de las necesidades humanas para las generaciones presentes y futuras. Levard y Marín (2000) plantean que en Nicaragua durante más de cuatro décadas se han desarrollados esfuerzos en la extensión con el propósito de mejorar las técnicas y métodos de producción de los agricultores.

Adopción de tecnologías agropecuarias. Una tecnología agropecuaria es el conjunto de conocimientos técnicos, científicamente ordenados, que permiten diseñar y crear bienes, servicios que facilitan la adaptación al medio ambiente y la satisfacción de las necesidades esenciales de las personas. Este concepto incluye: variedades, híbridos, razas de animales, sembradoras fertilizadoras, molinos picadores, maquina harinera, etc. (INTA, 2016).

En este estudio se identificaron 87 tecnologías aplicadas en los sistemas de producción, de este total 29 corresponden al uso de semilla mejorada de frijol, maíz, sorgo y arroz (Frijol INTA Sequia, Frijol INTA Rojo, Maíz H-INTA 991, Maíz NB-6, Maíz NB-9043, Frijol INTA Masatepe, Frijol Dor-364, Maíz Nutrinta amarillo, Maíz Nutrader, Frijol INTA canela, Frijol INTA Jinotega, Arroz INTA dorado, Frijol INTA Estelí, Frijol INTA Cárdenas, Frijol INTA Ferroso, Frijol Estelí 150, Arroz INTA Fortaleza, Frijol Estelí 50, Frijol Rojo Seda, Frijol T2, Maíz Mazorca de Oro, Frijol Estelí 90, Frijol INTA Matagalpa, Frijol INTA Nutritivo, Frijol INTA Norte, Frijol INTA Maravilla, Frijol L3, Sorgo Blanco, Sorgo Pinolero), nueve a variedades mejoradas de hortalizas (Ayote Carreta, Chiltoma Tres Cantos, Cebolla Sebaqueña, Tomate INTA Jinotega, Tomate JL-5, Tomate INTA Valle de Sébaco, Pipián Garza, Repollo Izalco, Repollo Escazú), seis en raíces y tubérculos (Papa Desiré, Papa Ona, Papa Granola, Papa Provento, Yuca Perla, Yuca Quintalera), 14 de pasturas (Pasto Brizantha, Caña de azúcar, Caña japonesa, Pasto CT-115, Pasto Gamba, Pasto Maralfalfa, Pasto Mulato, Pasto Mombaza, Pasto Marandú, Pasto Piatá, Pasto King Gras, Taiwán, Pasto Tanzania, Pasto Toledo), cinco de especies forrajeras (Cratylia, Leucaena, Gandul, Marango, Morera), 12 de manejo integrado de cultivo (Bacillus thuringiensis, biodigestor, Beauveria bassiana, bocashi, caldo bordelés, caldo sulfocálsico, neem para el manejo de garrapata, micorrizas, lombrihumos, sustrato, biofertilizante, micro túnel), cuatro de manejo de fuentes de agua (Laguneta, riego por goteo artesanal, reservorio, riego por aspersión artesanal), tres sobre alimentación en época seca para ganado mayor (bloque multinutricional, sal mineral, saccharina) y cinco en manejo post cosecha (caseta de secado, súper bolsa, silo metálico, troja mejorada, túnel de secado).

En el cuadro 2 se observa que las tecnologías de mayor adopción corresponden al uso de las semillas certificadas de frijol INTA Sequía y frijol INTA Rojo; como alternativa de fertilización el uso de biofertilizante; como parte del manejo post cosecha el uso de silo metálico; y el establecimiento de reservorio como tecnologías de cosecha de agua. 
La adopción de tecnología es muy variable, depende del grado de instrucción, de la experiencia previa, de la localidad, del sistema de producción, del costo que tiene la tecnología, de la complejidad de aplicación e inclusive, puede estar condicionada por cuestiones culturales, políticas y religiosas (Vicine, 2015).

Se evidenció que los aspectos sociales como la tenencia de la tierra, número de hijos, edad del productor y el nivel de escolaridad influyen directamente en una mayor o menor adopción de tecnologías agropecuarias. También se identificó que la adopción de tecnológica se ve influenciada por la diversidad productiva y cultural de cada municipio, así como por el acompañamiento de instituciones del estado que integran el Sistema Nacional de Producción, Consumo y Comercio, (incluye al INTA), universidades y organismos de cooperación internacional,

Cuadro 2. Tecnologías agropecuarias de mayor adopción en sistemas productivos de siete municipios de Jinotega

\begin{tabular}{lccccccc}
\hline \multirow{2}{*}{ Tecnologías* } & \multicolumn{7}{c}{ Porcentaje de productores por municipio } \\
\cline { 2 - 8 } & Jinotega & $\begin{array}{c}\text { San Rafael } \\
\text { del Norte }\end{array}$ & $\begin{array}{c}\text { La } \\
\text { Concordia }\end{array}$ & Yali** & Pantasma** & $\begin{array}{c}\text { El } \\
\text { Cuá }\end{array}$ & Wiwili \\
\hline Frijol INTA sequia & 87 & 82 & 100 & 91 & 75 & 37 & 55 \\
Frijol INTA rojo & 37 & 45 & 67 & 54 & 37 & 87 & 55 \\
Frijol Dor-364 & 13 & 9 & 11 & 36 & 25 & 25 & 11 \\
Maíz H-991 & 13 & 9 & 11 & 18 & 100 & 87 & 44 \\
Maíz NB-6 & 13 & 18 & 78 & 54 & 25 & 37 & 11 \\
Maíz NB-9043 & 25 & 0 & 11 & 72 & 0 & 62 & 33 \\
Maíz Nutrinta amarillo & 13 & 27 & 0 & 45 & 0 & 25 & 0 \\
Biofertilizante & 91 & 91 & 100 & 91 & 50 & 50 & 78 \\
Caldo sulfocalcico & 62 & 36 & 67 & 72 & 37 & 25 & 33 \\
Caldo bordelés & 13 & 0 & 33 & 36 & 13 & 13 & 11 \\
Silo metálico & 62 & 73 & 100 & 100 & 50 & 87 & 78 \\
Bloque multitrional & 62 & 36 & 33 & 18 & 25 & 25 & 33 \\
Sal mineral & 50 & 27 & 33 & 18 & 25 & 25 & 22 \\
Sacharina & 13 & 9 & 0 & 0 & 25 & 25 & 22 \\
Reservorio & 50 & 54 & 78 & 54 & 37 & 25 & 33 \\
\hline
\end{tabular}

* Tecnologías agropecuarias según INTA (2016) / ** Yalí: San Sebastián de Yalí, Pantasma: Santa María de Pantasma.

a través de estrategias colaborativas de trabajo como las planteadas en el Sistema Nicaragüense de Investigación e Innovación Agropecuaria (SNIA).

\section{Capacitaciones agropecuarias recibidas}

Capacitaciones agrícolas. Los productores expresan que las capacitaciones recibidas han estado orientadas al manejo agronómico de granos básicos (maíz, frijol, sorgo y arroz), hortalizas (chiltoma, tomate, cebolla y repollo), raíces y tubérculos (papa, yuca y malanga), así como al manejo post cosecha, manejo integrado de plagas y enfermedades, producción de semilla, agricultura orgánica y organización de bancos comunitarios de semillas.
En el cuadro 3 se observar que todos los productores han recibido capacitación sobre manejo agronómico de granos básicos, así como manejo y conservación de suelo y agua; sin embargo, la mayoría (63\%) también han sido capacitados en temas como manejo integrado de plagas, agricultura orgánica, manejo post cosecha de granos básicos y producción de semilla. El $26 \%$ corresponde al grupo de productores que han recibido entre siete y nueve capacitaciones, siendo este el conjunto con mayor cantidad de capacitaciones recibidas.

Las tecnologías y los procesos basados en el conocimiento son importantes porque permiten a los agricultores reaccionar de manera constructiva ante las dificultades, y aprovechar al máximo los beneficios (IICA, 2011). Orozco et al., (2009) indican que el aumentar el nivel de conocimientos en promotores, permite transmitir el conocimiento adquirido hacia los participantes en las comunidades de origen, aspecto trascendental en la adopción de prácticas y tecnologías agropecuarias.

Si los productores se capacitan constantemente en diversos temas agrícolas, son capases de aplicar y adoptar con más facilidad las prácticas y tecnologías aprendidas en los procesos de capacitación y además, pueden trasmitir ese conocimiento a los miembros de su familia y a otros productores de la comunidad.

Capacitaciones pecuarias. Se determinó que, del total de productores, el $17 \%$ no han participado en este tipo de capacitaciones, mientras que el $83 \%$ reportan que han recibido de una a cinco capacitaciones.

El mayor porcentaje de productores (64\%) indican que los temas de capacitación en los que han participado corresponden a manejo zoosanitario de ganado mayor y menor, manejo de pasturas mejoradas, alternativas de alimentación en época seca y sistemas silvopastoriles. Solo un $2 \%$ incluye además el registro zoosanitario.

Estos resultados revelan que no se ha trabajado activamente en el desarrollo de capacidades técnicas orientadas a la producción pecuaria, limitando la adopción de este tipo de prácticas y tecnologías en los sistemas de producción.

Según Salas et al., (2011), la adopción de tecnologías es un proceso que comienza con el primer conocimiento y termina cuando el individuo lo incorpora prácticamente, a su sistema de trabajo o a su modo de vida, con el fin de elevar la productividad de su unidad productiva. 
En este sentido el INTA promueve y desarrolla la generación y transferencia de tecnología para mejorar el manejo e incremento de los índices productivos y reproductivos de especies mayores y menores; esta transferencia está basada en el fortalecimiento continuo de conocimientos y capacidades técnicas, por medio de talleres, demostraciones prácticas y giras de intercambio con otros agricultores, utilizando como principal metodología la de aprender haciendo. daños a la finca, permitiéndole adquirir el conocimiento y habilidad práctica. Las giras de campo también son consideradas como un buen método de difusión de las tecnologías.

La estrategia de aprender haciendo es bien recibida por los productores, ya que alcanza un altos grado de aceptación de las tecnologías y prácticas que se promueven (IICA, 2010). En este sentido la demostración práctica tiene como objetivo principal mostrar de forma fácil

Cuadro 3. Temáticas de capacitación recibidas según número de productores

\begin{tabular}{clrrr}
\hline Capacitaciones & \multicolumn{1}{c}{ Temática } & Productores & Porcentaje & $\sum \%$ \\
\hline 2 & MAG, MCSA & 3 & 5 & \multirow{2}{*}{11} \\
3 & MAG, MCSA, MIC & 4 & 6 & \\
\cline { 2 - 6 } 4 & MAG, MCSA, MIC, AO & 14 & 22 & \\
5 & MAG, MCSA, MIC, AO, MPGB & 12 & 18 & 63 \\
6 & MAG, MCSA, MIC, AO, MPGB, PS, & 15 & 23 & \\
7 & MAG, MCSA, MIC, AO, MPGB, PS, MAH, & 8 & 12 & \\
8 & MAG, MCSA, MIC, AO, MPGB, PS, MAH, ECA & 6 & 9 & 26 \\
9 & MAG, MCSA, MIC, AO, MPGB, PS, MAH, ECA, BCS & 3 & 5 & \\
\hline Total & & 65 & 100 & 100 \\
\hline
\end{tabular}

MAG: manejo agronómico de granos básicos, MCSA: manejo y conservación de suelos y agua, MIC: manejo integrado de cultivo, AO: Agricultura orgánica, MPGB: manejo post cosecha de granos básicos, PS: producción de semilla, MAH: manejo agronómico de hortalizas, ECA: escuelas de campo, BCS: bancos comunitarios de semilla.

Métodos de difusión de tecnologías y prácticas agropecuarias. Los métodos de difusión de tecnologías juegan un rol importante ya que se implementan estrategias, técnicas y metodológicas que contribuyen a la adopción de prácticas y tecnologías a través de asistencia técnica, demostraciones prácticas, día de campo, escuelas de campo (ECA), giras de campo y talleres.

Delgado (2009), plantea que existen métodos individuales, grupales y masivos que realizan los extensionistas, para dar a conocer las tecnologías a los agricultores, induciéndolos a adoptarlas, incrementando así su bienestar social y económico.

En la figura 1 se observa que el mayor porcentaje de productores expresan que el método de demostración práctica es más eficiente en la adopción de tecnologías, debido a que los productores en conjunto con el técnico realizan el paso a paso en el establecimiento de una tecnología o en la implementación de una práctica, sobre todo cuando se utiliza la metodología de aprender haciendo. Esto ayuda a que los productores repliquen la práctica con sus familias y productores ale- la aplicación de ciertas técnicas, creando habilidades en los agricultores, quienes aprenden mediante la observación y la práctica.

\section{CONCLUSIONES}

A mayor nivel de escolaridad, menor edad del productor, mayor número de hijos que habitan en la finca y la tenencia de la tierra, se constituyen en factores que determinan mayoritariamente la aplicación y adopción de prácticas y tecnologías agropecuarias.

Se registraron 87 tecnologías y 18 prácticas agropecuarias. Las tecnologías de mayor adopción corresponden al uso de semilla certificada de frijol INTA sequía e INTA rojo, uso de biofertilizante, silos metálicos y el establecimiento de reservorios. En relación a las prácticas las de mayor aplicación y adopción son barreras vivas, barreras muertas, acequias, la no quema, rotación de cultivos, uso de neem más hoja de madero negro como bioinsumos, desparasitación, vacunación, vitaminización y ordeño limpio.

Las demostraciones prácticas y giras de campo son los mejores medios de difusión de la información y permiten mayor adopción de prácticas y tecnologías.

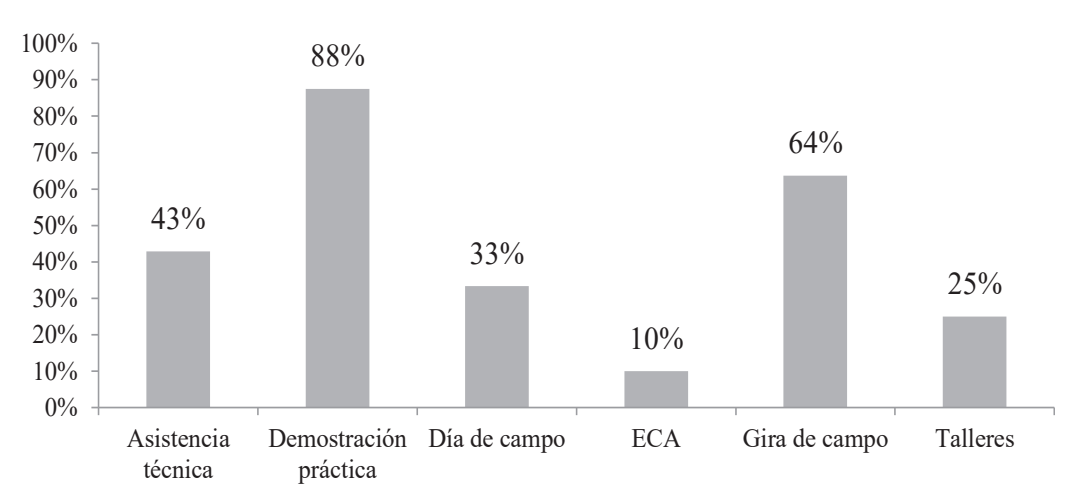

Figura 1. Métodos de difusión de tecnología agropecuarias implementados por el INTA. 


\section{AGRADECIMIENTO}

Los autores agradecen a los productores de Jinotega, San Rafael del Norte, La Concordia, San Sebastián de Yalí, Santa María de Pantasma, El Cuá y Wiwilí, así como a los técnicos del INTA delegación Jinotega, por hacer posible este trabajo de investigación. A los estudiantes de Agroecología de la UNAN-FAREM Jinotega que participaron en el estudio, al Instituto Nicaragüense de Tecnología Agropecuaria y a la Universidad Nacional Agraria por todo el apoyo brindado.

\section{REFERENCIAS BIBLIOGRÁFICAS}

Bernardo, J; Caldero, JF. 2000. Investigación cuantitativa (4): Métodos no experimentales. Aprendo a investigar en educación. Madrid: RIALP, SA. p. 77-93

Delgado Nagüera, W. 2009. Caracterización del proceso de transferencia y adopción tecnológica de pequeños y medianos productores de cebolla (Allium cepa L.) en el municipio de Pasca. (en línea). Consultado 15 dic. 2017. Disponible en http://www.javeriana.edu. co/biblos/tesis/eambientales/tesis12.pdf

FAO (Organización de las Naciones Unidas para la Alimentación y la Agricultura, IT). 2014. Agricultores familiares: Alimentar al mundo, cuidar el planeta. (en línea). Consultado 30 ene. 2018. Disponible en http://www.fao.org/fileadmin/user_upload/iyff/pdf/ Family_Farming_leaflet-print-es_01.pdf

2003. Tenencia de la tierra y desarrollo rural. (en línea). Consultado 15 dic. 2017. Disponible en http://www.fao.org/docrep/005/Y4307S/Y4307S00.HTM

1998. Evaluación de la sostenibilidad para la agricultura y la alimentación (SAFA). (en línea). Consultado 4 sep. 2017. Disponible en http://www.fao.org/nr/sustainability/evaluaciones-de-la-sostenibilidad-safa/es/

Gamboa, M; Vanderschaeghe, M. 2013. Perfil de género en la economía de Jinotega fundación ETEA-ONU Mujeres. (en línea). Consultado 15 nov. 2017. Disponible en http://www.fundacionetea.org/media/File/Perfi_genero_\%20economia_\%20jinotega.pdf.pdf

Greig, L. 2009. An analysis of the key factors influencing farmer's choice of crop kibamba ward, Tanzania. J Agric Econ (60):699-715.

IICA (Instituto Interamericano de Cooperación para la Agricultura, CO). 2011. Análisis del extensionismo agrícola en México. (en línea). Consultado 3 nov. 2017. Disponible en http://www.sagarpa.gob.mx/desarrolloRural/DesCap/Documents/AnalisisExtensionismoAgricolaMexico.pdf

2010. Escuelas de campo para agricultores de Café y Cacao. (en línea). Consultado 3 nov. 2017. Disponible en file://C:/ Users/ELITEDESK/Desktop/A5997E.PDF

INTA (Instituto Nicaragüense de Tecnología Agropecuaria). 2016. Norma técnica de transferencia de tecnología.

2015. Catálogo de tecnologías para enfrentar el cambio climático. (en línea). Consultado 26 ene. 2017. Disponible en http:// www.inta.gob.ni/index.php/biblioteca-virtual/catalogo-de-tecnologias.

Levard, L; Marin, Y. 2000. La problemática técnica y la intervención de los organismos que trabajan en el fomento tecnológico en el trópico seco de Nicaragua. (en linea). Consultado 12 ene. 2018. Disponible en http://www.uca.edu.ni/2/images/Revista-Encuentro/ Revistas/e53/art-1.pdf

Münch, G; Ángeles, E. 1996. Métodos y técnicas de investigación para administración e ingeniería. 2 ed. Trillas, MX. 166 p.

MAG (Ministerio Agropecuario, NI). 2012. Cuarto censo nacional agropecuario CENAGRO. (en línea). Consultado 5 dic. 2015. Disponible en http://www.magfor.gob.ni/prorural/IIMesa2012/Presentacion\%20IV\%20CENAGRO.pdf

Orozco, C; Ramírez Valverde, B; Flores, A; Jiménez Sánchez, L; Estrella Chulim, N; Peña Olvera, BV; Ramos Sánchez, A; Morales Guerra, M. 2009. Impacto del conocimiento tecnológico sobre la adopción de tecnología agrícola en campesinos indígena de México. (en línea). Consultado 4 ago. 2015. Disponible en http://www.scielo.org.ve/pdf/inci/v34n8/art07.pdf

Pineda Rizo, OM; Guzmán Guillen, F. 2015. Adopción de tecnologías de conservación de suelos y agua y su efecto en los ingresos agropecuarios y el contenido de materia orgánica del suelo. La Calera 15(25):77-81. Fuente original: Nowak, p. 1992. Why farmer adopt production technology. Journal of Soil and Water conservation. 47(1):14-16.

Roco, L; Engler, A; Jará, R. 2012. Factores que influyen en la adopción de tecnologías de conservación de suelos en el secano interior de Chile Central. (en línea). Consultado 5 dic. 2017. Disponible en http://www.scielo.org.ar/img/revistas/refca/v44n $2 / \mathrm{html} / \mathrm{v} 44 \mathrm{n} 2 \mathrm{a} 03 . \mathrm{htm}$

Rojas, J. 2015. Medición de la innovación agropecuaria desde los territorios: una propuesta conceptual y metodológica. (en línea). Consultado 12 ene. 2018. Disponible en http://repositorio.una.edu.ni/3374/1/ppc20r741.pdf

Salas González, J; Leos Rodríguez, J; Sagarnaga Villegas, L; Zavala Pineda, M. 2011. Adopción de tecnologías por productores beneficiarios del programa de estímulos a la productividad ganadera (PROGAN) en México. (en línea). Consultado 29 nov. 2017. Disponible en http://www.scielo.org.mx/scielo.php?script=sci_arttext\&pid=S2007-11242013000200010

Vicine, L. 2015. Adopción de tecnologías agrícolas. (en línea). Consultado 2 ago. 2017. Disponible en http://inta.gob.ar/sites/default/ files/script-tmp-adopcion_tecnologia_agricola.pdf 